\title{
Corrigendum: Tissue engineering of acellular vascular grafts capable of somatic growth in young lambs
}

Zeeshan Syedain, Jay Reimer, Matthew Lahti, James Berry, Sandra Johnson, Richard Bianco \& Robert T. Tranquillo

Nature Communications 7:12951 doi:10.1038/ncomms12951 (2016); Published 27 Sept 2016; Updated 17 Jan 2017

The authors inadvertently omitted Richard Bianco, who provided consultation for the experimental surgical services, from the author list. This has now been corrected in both the PDF and HTML versions of the Article. 